\title{
McArdle disease: a case report and review
}

This article was published in the following Dove Press journal:

International Medical Case Reports Journal

19 January 2012

Number of times this article has been viewed

\section{Alberto Leite \\ Narciso Oliveira \\ Manuela Rocha \\ Internal Medicine Department, Hospital de Braga, Portugal}

Correspondence: Alberto Leite Internal Medicine Department, Hospital de Braga, Sete Fontes - São Vitor, 47I0-243 Braga, Portugal Tel +35 I9I 5301699

Email alberto_leite@sapo.pt
Abstract: McArdle disease (glycogen storage disease type V) is a pure myopathy caused by an inherited deficit of myophosphorylase. The disease exhibits clinical heterogeneity, but patients typically experience exercise intolerance, acute crises of early fatigue, and contractures, sometimes with rhabdomyolysis and myoglobinuria, triggered by static muscle contractions or dynamic exercise. We present the case of a 54-year-old man with a lifelong history of fatigability, worsening on exertion. Laboratory evaluation revealed significant elevations in levels of creatine kinase (7924 U/L), lactate dehydrogenase (624 U/L), and myoglobulin $(671 \mathrm{ng} / \mathrm{mL})$. A muscle biopsy confirmed the presence of McArdle disease. This case report illustrates how, due to embarrassment, the patient hid his symptoms for many years and was eventually extremely relieved and "liberated" once McArdle disease was diagnosed 40 years later.

Keywords: McArdle disease, glycogen storage disease, myophosphorylase

\section{Introduction}

Glycogenosis type V, also known as glycogen storage disease type V (GSD V), myophosphorylase deficiency, or McArdle disease (Online Mendelian Inheritance in $\operatorname{Man}^{\circledR}$ [OMIM ${ }^{\circledR}$; Johns Hopkins University, Baltimore, MD] number 232600), is a rare, mostly autosomal recessive disorder causing deficient myophosphorylation of glycogen in the skeletal muscles. ${ }^{1}$ Patients with McArdle disease have mutations in both alleles of the PYGM gene, which encodes myophosphorylase, the skeletal muscle isoform of glycogen phosphorylase. ${ }^{2}$ As the liver and heart isoforms of glycogen phosphorylase are unaffected, McArdle disease presents as a pure myopathy. ${ }^{3}$ Here, we report the case of a 54-year-old man with McArdle disease who had a long history of fatigability and exercise intolerance; he became unhappy and depressed because of not having an explanation for his symptoms for most of his life. We also summarize the main features of McArdle disease, including diagnostic tools and current therapeutic options.

\section{Case report}

A 54-year-old man presented to our service with a lifelong history of fatigability that worsened on exertion. Even as a child, he had experienced difficulty going up hills and lagged behind when he went out for a walk with friends. Since his childhood, he had been labeled as "lazy" by family and friends. He consulted several doctors, who were unable to provide an explanation for his symptoms, adding to his unhappiness and resulting in depression. He presented to our service with early fatigue complaints 
and contractures triggered by dynamic exercise (climbing stairs) during a visit to his mother in hospital. He described a sensation of his legs becoming stiff like a board after walking for a while; this symptom was relieved by rest. These symptoms were worse if he was hungry and were alleviated after meals. There were no diurnal fluctuations in symptoms, and findings of physical examination were unremarkable. There was no neuromuscular disease in his family history. He had depression and a long history of alcohol abuse. He had been receiving long-term treatment with tianeptine, bromazepam, omeprazole, and silymarin. At presentation, results of routine blood and chemistry tests showed a creatine kinase (CK) level of $7924 \mathrm{U} / \mathrm{L}$, a lactate dehydrogenase level of $624 \mathrm{U} / \mathrm{L}$, and a myoglobulin level of $671 \mathrm{ng} / \mathrm{mL}$. A repeat check performed after cessation of treatment revealed a CK level of 2945 U/L. There was no myoglobinuria. Results of tests for autoimmune disease-related antibodies such as antinuclear antibody and anti-SSA, anti-SSB, and Topo-I antibodies were negative. Needle electromyography findings were normal at rest but upon volunteer effort, showed a myopathic pattern characterized by reduced duration and amplitude, and increased recruitment of potential short polyphasic motor units in the biceps and deltoid muscles. A test of sensory nerve conduction showed normal findings. A muscle biopsy specimen stained for glycogen showed subsarcolemmal accumulation of "lakes" of glycogen. Results of enzyme histochemistry were completely negative for myophosphorylase, confirming the diagnosis of McArdle disease (GSD V). A genetic study showed double heterozygosity of the PYGM gene, with the mutation c.148C $>\mathrm{T}$ (p.R49X) in exon 1 and the change c. $345+1 \mathrm{G}>\mathrm{A}$ in intron 2 , which, according to the bioinformatics simulations carried out, is a splicing mutation.

\section{Discussion}

Myophosphorylase initiates the breakdown of muscle glycogen by removing $(1,4)$ - $\alpha$-glucosyl units from the outer branches of glycogen, leading to liberation of glucose-1-phosphate, which subsequently undergoes glycolysis. As a consequence of deficient myophosphorylase activity, patients with McArdle disease are unable to obtain energy from their muscle glycogen stores. ${ }^{4}$ Given that glycolysis is blocked upstream, the skeletal muscle fibers of patients with McArdle disease can still take up glucose from the blood and convert it into glucose-6-phosphate, which then enters the downstream steps of glycolysis. Therefore, muscle glycolysis is not totally impaired in these patients, and preexercise ingestion of carbohydrates can markedly improve their exercise tolerance..$^{5}$ Acute exercise can trigger episodes of reversible "muscle crises" that manifest mainly in the form of excessive, premature fatigue and contractures, frequently accompanied by marked muscle breakdown (rhabdomyolysis) and sometimes by myoglobinuria. Potential differential diagnosis includes defects in phosphofructokinase (PFK; GSD VII or Tarui disease), phosphorylase $b$ kinase (GSD VIII), phosphoglycerate kinase (GSD IX), phosphoglyceromutase (GSD X), lactate dehydrogenase (GSD XI), and $\beta$-enolase (GSD XIII).

There is individual variability in the clinical manifestation of McArdle disease. The time of onset of the typical disease symptoms and the degree of exercise intolerance vary between patients. One reason for this clinical heterogeneity might be different dietary regimes and lifestyles. Another fact that might contribute to phenotypic heterogeneity is the patient's gender, with women generally being more severely affected than men. ${ }^{6,7}$ Some patients can be oligosymptomatic, but in rare cases the disease can be profoundly incapacitating. Disease severity has been correlated with genotype at the angiotensin-converting enzyme (ACE) locus, where an insertion/deletion variant (the D allele) associated with modestly increased ACE activity is found more commonly in patients with more severe symptoms and reduced exercise tolerance. ${ }^{8}$ McArdle disease is not usually a lifethreatening condition, although there are some exceptions. Myoglobinuria occurs in about $50 \%$ of patients after intense exercise and about $50 \%$ of these patients also develop acute renal failure, which is almost always reversible but requires emergency treatment. ${ }^{9}$

The main diagnostic tools for McArdle disease are four features present in the majority of patients. The first is exercise intolerance, usually present from childhood, with or without actual fixed muscle weakness at the time of evaluation. The patient might also report improved exercise tolerance after ingesting carbohydrates. Another classic feature is a high serum level of total CK activity, even at rest, in the absence of heavy exercise in the previous hours or days. A third characteristic of the disease is one or more previous episodes of elevated CK serum levels after intense exercise. The fourth feature is the "second wind" phenomenon, a pathognomonic feature of McArdle disease. ${ }^{10}$ First described by Pearson et al, the second wind phenomenon denotes a sudden, marked improvement in the tolerance to aerobic, dynamic, large muscle mass exercise after approximately 10 minutes, that is, the disappearance of the excessive fatigue, breathlessness, and tachycardia that are triggered at the start of exertion. ${ }^{11}$ Most patients describe this phenomenon as the ability to resume exercising if they take a brief rest at the appearance 
of premature fatigue early during exercise. This second wind can be explained by increased blood flow, enhanced delivery of free fatty acids with concurrent activation of fatty acid metabolism, and increased glucose utilization. In fact, Haller and Vissing showed that the second wind phenomenon is abolished by glucose infusion or sucrose ingestion before exercise. ${ }^{12}$

The first diagnostic test used for McArdle disease was a forearm ischemic test, but this can be painful and can produce false-negative results in the weakest patients or false-positive results in patients with other defects of glycogenolysis or glycolysis. Biopsy of the vastus lateralis or biceps brachialis muscles is commonly conducted in patients with McArdle disease, in order to enable histochemical and biochemical analysis. In addition to subsarcolemmal or intermyofibrillar glycogen deposits, muscle specimens from patients with McArdle disease show a negative histochemical reaction for myophosphorylase and null activity of this enzyme. ${ }^{13}$ Diagnosis based on molecular genetics can be very useful, where the required technology is available. Around 100 different mutations have been described in PYGM. The number of mutations continues to increase, highlighting the importance of complementary DNA-based molecular studies for the diagnosis of McArdle disease. ${ }^{14}$

Despite all efforts, no effective gene therapy is available to alleviate myophosphorylase deficiency in humans. ${ }^{15,16} \mathrm{At}$ present, the most beneficial intervention for patients with McArdle disease, in combination with aerobic conditioning, is ensuring that sufficient blood glucose is constantly available to patients' working muscles during the daytime. This is achieved by adopting a diet with a high proportion $(65 \%)$ of complex carbohydrates (such as those found in vegetables, fruits, cereals, bread, pasta, and rice) and a low proportion $(20 \%)$ of fat. In addition, the ingestion of simple carbohydrates (20-40 g of glucose or fructose in adults and $20 \mathrm{~g}$ in children) approximately 5 minutes before engaging in strenuous exercise, such as brisk walking or physical education in younger patients, can be helpful. In general, vigorous dynamic exercise should be performed only by the more fit patients and should be combined with pre-exercise carbohydrate ingestion. Very intense exercises, particularly those involving high loads on low muscle mass, are strongly discouraged in these patients. Increasing copies of the D allele at the ACE locus is associated with disease severity, especially in women; the preliminary results of a study based on this observation suggest that treatment with the ACE inhibitor ramipril is beneficial. ${ }^{17}$

\section{Conclusion}

McArdle disease is a pure myopathy caused by an inherited deficit of the skeletal muscle isoform of glycogen phosphorylase. This syndrome is characterized by exercise intolerance, with most patients experiencing acute "muscle crises" after static or intense dynamic exercise. Crises appear in the form of early muscle fatigue and contractures, sometimes with marked muscle breakdown (rhabdomyolysis) and myoglobinuria. No effective gene therapy is expected to be available in the short term. Until a definitive cure is found, patients can benefit from adopting a diet rich in complex carbohydrates and ingesting simple carbohydrates before strenuous exercise. Carefully supervised, regular, aerobic exercise of low-to-moderate intensity is a safe, recommended therapeutic option, but exercises that involve heavy static contractions or induce severe myalgia should be avoided. The benefits of creatine supplementation, treatment with ACE inhibitors, and vitamin B6 supplementation remain unproven for patients with muscle phosphorylase deficiency. ${ }^{18}$

\section{Disclosure}

The authors have no conflicts of interest in this work.

\section{References}

1. Haller RG. Treatment of McArdle disease. Arch Neurol. 2000;57: 923-924.

2. Andreu AL, Nogales-Gadea G, Cassandrini D, Arenas J, Bruno C. McArdle disease: molecular genetic update. Acta Myol. 2007;26(1):53-57.

3. DiMauro S. Muscle glycogenoses: an overview. Acta Myol. 2007;26(1):35-41.

4. Dimaur S, Andreu AL, Bruno C, Hadjigeorgiou GM. Myophosphorylase deficiency (glycogenosis type V; McArdle disease). Curr Mol Med. 2002;2(2):189-196.

5. Vissing J, Haller RG. The effect of oral sucrose on exercise tolerance in patients with McArdle's disease. $N$ Eng J Med. 2003;349(26):2503-2509.

6. Rubio JC, Gómez-Gallego F, Santiago C, et al. Genotype modulators of clinical severity in McArdle disease. Neurosci Lett. 2007; 422(3):217-222.

7. Rommel O, Kley RA, Dekomien G, Epplen JT, Vorgerd M, Hasenbring M. Muscle pain in myophosphorylase deficiency (McArdle's disease): the role of gender, genotype, and pain-related coping. Pain. 2006;124(3):295-304.

8. Martinuzzi A, Sartori E, Fanin M, et al. Phenotype modulators in myophosphorylase deficiency. Ann Neurol. 2003;53(4):497-502.

9. DiMauro S, Tsujlino S. Nonlysosomal glycogenoses. In: Engel AG, Franzini-Armstrong C, editors. Myology. 2nd ed. New York, NY: McGraw Hill; 1994:1554-1556.

10. Lucia A, Nogales-Gadea G, Pérez M, Martin MA, Andreu AL, Arenas J. McArdle disease: what do neurologists need to know? Nat Clin Prac. 2008;4(10):568-577.

11. Pearson CM, Rimer DG, Mommaerts WF. A metabolic myopathy due to absence of muscle phosphorylase. Am J Med. 1961;30:502-517.

12. Haller RG, Vissing J. Spontaneous "second wind" and glucose-induced second "second wind" in McArdle disease: oxidative mechanisms. Arch Neurol. 2002;59(9):1395-1402. 
13. Nogales-Gadea G, Arenas J, Andreu AL. Molecular genetics of McArdle's disease. Curr Neurol Neurosci Resp. 2007;7(1):84-92.

14. Fernandez-Cadenas I, Andreu AL, Gamez J, et al. Splicing mosaic of the myophosphorylase gene due to a silent mutation in McArdle disease. Neurology. 2003;61(10):1432-1434.

15. Pari G, Crerar MM, Nalbantoglu J, et al. Myophosphorylase gene transfer in McArdle's disease myoblasts in vitro. Neurology. 1999;53(6):1352-1354.

16. Howeel JM, Walker KR, Davies L, et al. Adenovirus and adenoassociated virus-mediated delivery of human myophosphorylase cDNA and LacZ cDNA to muscle in the ovine model of McArdle's disease: expression and re-expression of glycogen phosphorylase. Neuromuscul Disord. 2008;18(3):248-258.
17. Martinuzzi A, Liava A, Trevisi E, et al. Randomized, placebocontrolled, double-blind pilot trial of ramipril in McArdle's disease. Muscle Nerve. 2008;37(3):350-357.

18. Kyel RA, Tarnopolsky MA, Vorgerd M. Creatine for treating muscle disorders. Cochrane Database Syst Rev. 2011;2:CD004760.

\section{Publish your work in this journal}

The International Medical Case Reports Journal is an international, peer-reviewed open-access journal publishing original case reports from all medical specialties. Previously unpublished medical posters are also accepted relating to any area of clinical or preclinical science. Submissions should not normally exceed 2,000 words or
4 published pages including figures, diagrams and references. The manuscript management system is completely online and includes a very quick and fair peer-review system, which is all easy to use. Visit http://www.dovepress.com/testimonials.php to read real quotes from published authors.

Submit your manuscript here: http://www.dovepress.com/international-medical-case-reports-journal-journal 